\title{
2-LOCAL DERIVATIONS ON INFINITE-DIMENSIONAL LIE ALGEBRAS
}

\author{
SHAVKAT AYUPOV ${ }^{1,2}$, BAXTIYOR YUSUPOV ${ }^{3}$
}

\begin{abstract}
The present paper is devoted to study 2-local derivations on infinitedimensional Lie algebras over a field of characteristic zero. We prove that all 2-local derivations on the Witt algebra as well as on the positive Witt algebra are (global) derivations, and give an example of infinite-dimensional Lie algebra with a 2-local derivation which is not a derivation.

Keywords: Lie algebras, Witt algebra, positive Witt algebra, thin Lie algebra, derivation, 2-local derivation.
\end{abstract}

AMS Subject Classification: 17A32, 17B30, 17B10.

\section{INTRODUCTION}

In 1997, Šemrl 8 introduced the notion of 2-local derivations and 2-local automorphisms on algebras. Namely, a map $\Delta: \mathcal{L} \rightarrow \mathcal{L}$ (not necessarily linear) on an algebra $\mathcal{L}$ is called a 2-local derivation if, for every pair of elements $x, y \in \mathcal{L}$, there exists a derivation $D_{x, y}: \mathcal{L} \rightarrow \mathcal{L}$ such that $D_{x, y}(x)=\Delta(x)$ and $D_{x, y}(y)=\Delta(y)$. The notion of 2-local automorphism is given in a similar way. For a given algebra $\mathcal{L}$, the main problem concerning these notions is to prove that they automatically become a derivation (respectively, an automorphism) or to give examples of local and 2-local derivations or automorphisms of $\mathcal{L}$, which are not derivations or automorphisms, respectively. Solution of such problems for finite-dimensional Lie algebras over algebraically closed field of zero characteristic were obtained in [1,2] and [3]. Namely, in [2] it is proved that every 2-local derivation on a semi-simple Lie algebra $\mathcal{L}$ is a derivation and that each finite-dimensional nilpotent Lie algebra, with dimension larger than two admits 2-local derivation which is not a derivation. Concerning 2-local automorphism, Chen and Wang in [3] prove that if $\mathcal{L}$, is a simple Lie algebra of type $A_{l}, D_{l}$ or $E_{k},(k=6,7,8)$ over an algebraically closed field of characteristic zero, then every 2-local automorphism of $\mathcal{L}$, is an automorphism. Finally, in [1] Ayupov and Kudaybergenov generalized this result of 3 and proved that every 2-local automorphism of a finite-dimensional semi-simple Lie algebra over an algebraically closed field of characteristic zero is an automorphism. Moreover, they show also that every nilpotent Lie algebra with finite dimension larger than two admits 2-local automorphisms which is not an automorphism.

In the present paper we study 2-local derivations on infinite-dimensional Lie algebras over a field of characteristic zero.

In Section 2 we give some preliminaries concerning Witt and positive Witt algebras. In Section 3 we give a general form of derivations on the positive Witt algebra. In Section 4 we prove that every 2-local derivations on Witt algebra and on the positive Witt algebra are automatically derivations. We also show that so-called thin Lie algebras admit 2-local derivations which are not derivations. 


\section{Preliminaries}

In this section we give some necessary definitions and preliminary results.

A derivation on a Lie algebra $\mathcal{L}$ is a linear map $D: \mathcal{L} \rightarrow \mathcal{L}$ which satisfies the Leibniz law, that is,

$$
D([x, y])=[D(x), y]+[x, D(y)]
$$

for all $x, y \in \mathcal{L}$. The set of all derivations of $\mathcal{L}$ with respect to the commutation operation is a Lie algebra and it is denoted by $\operatorname{Der}(\mathcal{L})$. For all $a \in \mathcal{L}$, the map $a d(a)$ on $\mathcal{L}$ defined as $a d(a) x=[a, x], x \in \mathcal{L}$ is a derivation and derivations of this form are called inner derivation. The set of all inner derivations of $\mathcal{L}$, denoted $\operatorname{ad}(\mathcal{L})$, is an ideal in $\operatorname{Der}(\mathcal{L})$.

Let $A=\mathbb{C}\left[x, x^{-1}\right]$ be the algebra of all Laurent polynomials in one variable over a field of characteristic zero $\mathbb{F}$. The Lie algebra of derivations

$$
\operatorname{Der}(A)=\operatorname{span}\left\{f(x) \frac{d}{d x}: f \in \mathbb{C}\left[x, x^{-1}\right]\right\}
$$

with the Lie bracket is called a Witt algebra and denoted by $W$. Then [4] $W$ is an infinite-dimensional simple algebra which has the basis $\left\{e_{i}: e_{i}=x^{i+1} \frac{d}{d x}, i \in \mathbb{Z}\right\}$ and the multiplication rule

$$
\left[e_{i}, e_{j}\right]=(j-i) e_{i+j}, i, j \in \mathbb{Z}
$$

We also consider the infinite-dimensional positive part $W^{+}$of the Witt algebra. The positive Witt algebra $W^{+}$is an infinite-dimensional Lie algebra [7] which has the basis $\left\{e_{i}: e_{i}=x^{i+1} \frac{d}{d x}, i \in \mathbb{N}\right\}$ and the multiplication rule

$$
\left[e_{i}, e_{j}\right]=(j-i) e_{i+j}, i, j \in \mathbb{N} .
$$

Recall that a map $\Delta: \mathcal{L} \rightarrow \mathcal{L}$ (not liner in general) is called a 2-local derivation if for every $x, y \in \mathcal{L}$, there exists a derivation $D_{x, y}: \mathcal{L} \rightarrow \mathcal{L}$ (depending on $x, y$ ) such that $\Delta(x)=D_{x, y}(x)$ and $\Delta(x)=D_{x, y}(y)$.

Since any derivation on the infinite-dimensional Witt algebra $W$ is inner [6], it follows that for this algebra the above definition of the 2-local derivation can be reformulated as follows. A map $\Delta$ on $W$ is called a 2-local derivation on $W$, if for any two elements $x, y \in W$ there exists an element $a_{x, y} \in W$ (depending on $x, y$ ) such that

$$
\Delta(x)=\left[a_{x, y}, x\right], \Delta(y)=\left[a_{x, y}, y\right] .
$$

Henceforth, given a 2-local derivation on $W$, the symbol $a_{x, y}$ will denote the element from $W$ satisfying $\Delta(x)=\left[a_{x, y}, x\right]$ and $\Delta(y)=\left[a_{x, y}, y\right]$.

\section{Derivations on the Positive Witt algebra}

Let us consider the following algebra $W^{+}+\left\langle e_{0}\right\rangle=\operatorname{span}\left\{e_{n}: n=0,1,2, \ldots\right\}$ with the multiplication rule

$$
\left[e_{n}, e_{m}\right]=(m-n) e_{n+m}, \quad n, m \geq 0 .
$$

It is clear that $W^{+}$is an ideal in $W^{+}+\left\langle e_{0}\right\rangle$. Hence, any element $a \in W^{+}+\left\langle e_{0}\right\rangle$ defines a spatial derivation $L_{a}$ on $W^{+}$by the following way:

$$
L_{a}(x)=[a, x], \quad x \in W^{+} .
$$

Theorem 3.1. Let $D$ be a derivation on $W^{+}$. Then there exists an element $a \in W^{+}+$ $\left\langle e_{0}\right\rangle$ such that

$$
D=L_{a} .
$$


Proof. Let $D\left(e_{1}\right)=\alpha_{1} e_{1}+\alpha_{2} e_{2}+\ldots+\alpha_{n} e_{n}$. Take an element

$$
a_{1}=-\alpha_{3} e_{2}-\frac{\alpha_{4}}{2} e_{3}-\ldots-\frac{\alpha_{n}}{n-2} e_{n-1} .
$$

Then

$$
L_{a_{1}}\left(e_{1}\right)=\left[a_{1}, e_{1}\right]=\alpha_{3} e_{3}+\alpha_{4} e_{4}+\ldots+\alpha_{n} e_{n}=D\left(e_{1}\right)-\alpha_{1} e_{1}-\alpha_{2} e_{2} .
$$

Setting $D_{1}=D-L_{a_{1}}$, we have a derivation $D_{1}$ such that $D_{1}\left(e_{1}\right)=\alpha_{1} e_{1}+\alpha_{2} e_{2}$.

Now we shall show that $\alpha_{2}=0$. Suppose that $D_{1}\left(e_{2}\right)=\sum_{k \geq 1} \beta_{k} e_{k}$. Then

$$
\begin{aligned}
D_{1}\left(e_{3}\right) & =D_{1}\left(\left[e_{1}, e_{2}\right]\right)=\left[D_{1}\left(e_{1}\right), e_{2}\right]+\left[e_{1}, D_{1}\left(e_{2}\right)\right]= \\
& =\left(\alpha_{1}+\beta_{2}\right) e_{3}+\sum_{k \geq 2} k \beta_{k+1} e_{k+2}, \\
D_{1}\left(e_{4}\right) & =\frac{1}{2} D_{1}\left(\left[e_{1}, e_{3}\right]\right)=\frac{1}{2}\left[D_{1}\left(e_{1}\right), e_{3}\right]+\frac{1}{2}\left[e_{1}, D_{1}\left(e_{3}\right)\right]= \\
& =\left(2 \alpha_{1}+\beta_{2}\right) e_{4}+\frac{1}{2}\left(\alpha_{2}+6 \beta_{3}\right) e_{5}+\frac{1}{2} \sum_{k \geq 3}\left(k^{2}+k\right) \beta_{k+1} e_{k+3}, \\
D_{1}\left(e_{5}\right) & =\frac{1}{3} D_{1}\left(\left[e_{1}, e_{4}\right]\right)=\frac{1}{3}\left[D_{1}\left(e_{1}\right), e_{4}\right]+\frac{1}{3}\left[e_{1}, D_{1}\left(e_{4}\right)\right]= \\
& =\left(3 \alpha_{1}+\beta_{2}\right) e_{5}+\frac{1}{3}\left(4 \alpha_{2}+12 \beta_{3}\right) e_{6}+\frac{1}{6} \sum_{k \geq 3}\left(k^{3}+3 k^{2}+2 k\right) \beta_{k+1} e_{k+4}, \\
D_{1}\left(e_{5}\right) & =D_{1}\left(\left[e_{2}, e_{3}\right]\right)=\left[D_{1}\left(e_{2}\right), e_{3}\right]+\left[e_{2}, D_{1}\left(e_{3}\right)\right]= \\
& =2 \beta_{1} e_{4}+\left(\alpha_{1}+2 \beta_{2}\right) e_{5}+4 \beta_{3} e_{6}+\sum_{k \geq 3}\left(k^{2}-k+2\right) \beta_{k+1} e_{k+4} .
\end{aligned}
$$

Comparing the last two equalities we obtain that

$$
\alpha_{2}=\beta_{1}=0, \beta_{2}=2 \alpha_{1} \text { and } \beta_{k}=0, k \geq 4 .
$$

Therefore

$$
D_{1}\left(e_{1}\right)=\alpha_{1} e_{1} \text { and } D_{1}\left(e_{2}\right)=2 \alpha_{1} e_{2}+\beta_{3} e_{3} .
$$

Set $a_{2}=\alpha_{1} e_{0}$. Then

$$
L_{a_{2}}\left(e_{1}\right)=\left[\alpha_{1} e_{0}, e_{1}\right]=\alpha_{1} e_{1} .
$$

Setting $D_{2}=D_{1}-L_{a_{2}}$, we have a derivation $D_{2}$ such that $D_{2}\left(e_{1}\right)=0$.

Now by induction we shall show that

$$
D_{2}\left(e_{k}\right)=(k-1) \beta_{3} e_{k+1}, \text { for all } k \geq 2 .
$$

We have

$$
D_{2}\left(e_{2}\right)=D_{1}\left(e_{2}\right)-L_{a_{2}}\left(e_{2}\right)=2 \alpha_{1} e_{2}+\beta_{3} e_{3}-2 \alpha_{1} e_{2}=\beta_{3} e_{3} .
$$

Assume that

$$
D_{2}\left(e_{k}\right)=(k-1) \beta_{k} e_{k+1} .
$$

Then

$$
D_{2}\left(e_{k+1}\right)=\frac{1}{k-1} D_{2}\left(\left[e_{1}, e_{k}\right]\right)=\frac{1}{k-1}\left[D_{2}\left(e_{1}\right), e_{k}\right]+\frac{1}{k-1}\left[e_{1}, D_{2}\left(e_{k}\right)\right]=k \beta_{3} e_{k+2} .
$$

So

$$
D_{2}\left(e_{k}\right)=(k-1) \beta_{3} e_{k+1}, \text { for all } k \geq 2 \text {. }
$$


Take the element $a_{3}=\beta_{3} e_{1}$, then

$$
L_{a_{3}}\left(e_{k}\right)=\left[a_{3}, e_{i}\right]=\left[\beta_{3} e_{1}, e_{k}\right]=(k-1) \beta_{3} e_{k+1}, \quad k \geq 2 .
$$

This means that $D_{2}=L_{a_{3}}$. Thus

$$
D=D_{1}+L_{a_{1}}=D_{2}+L_{a_{1}}+L_{a_{2}}=L_{a_{3}}+L_{a_{2}}+L_{a_{3}}=L_{a},
$$

where $a=a_{1}+a_{2}+a_{3}$. The proof is complete.

Remark 3.2. Let $D=L_{a}$ be a derivation of the form (3.1), where $a=-\sum_{i=0}^{n} \alpha_{i} e_{i}$. Direct computations show that

$$
D\left(e_{j}\right)=\sum_{i=1}^{n} \alpha_{i}(j+1-i) e_{i+j-1}, \quad j \geq 1 .
$$

\section{2-LOCAL DERIVATIONS ON SOME INFINITE-DIMENSIONAL LIE ALGEBRAS}

Now we shall give the main result concerning 2-local derivations on infinitedimensional Lie algebras.

\subsection{2-Local derivations on the Witt algebra.}

Theorem 4.1. Let $W$ be the Witt algebra over a field of characteristic zero. Then any 2-local derivation on $W$ is a derivation.

For the proof of this Theorem we need several Lemmas.

Lemma 4.2. Let $\Delta$ be a 2-local derivation on $W$ such that

$$
\Delta\left(e_{0}\right)=\Delta\left(e_{1}\right)=0 .
$$

Then $\Delta\left(e_{i}\right)=0$ for all $i \in \mathbb{Z}$.

Proof. Let $i \in \mathbb{Z}$ be a fixed index except 0,1 . There exists an element $a_{e_{0}, e_{i}} \in W$ such that

Then

$$
\Delta\left(e_{0}\right)=\left[a_{e_{0}, e_{i}}, e_{0}\right], \quad \Delta\left(e_{i}\right)=\left[a_{e_{0}, e_{i}}, e_{i}\right]
$$

$$
0=\Delta\left(e_{0}\right)=\left[a_{e_{0}, e_{i}}, e_{0}\right]=\left[\sum_{j \in \mathbb{Z}} \alpha_{j} e_{j}, e_{0}\right]=\sum_{j \in \mathbb{Z}} \alpha_{j} j e_{j} .
$$

Thus $\alpha_{j}=0$ for all $j \in \mathbb{Z}$ with $j \neq 0$. This means that $a_{e_{0}, e_{i}}=\alpha_{0} e_{0}$. Thus

$$
\Delta\left(e_{i}\right)=\left[a_{e_{0}, e_{i}}, e_{i}\right]=\left[\alpha_{0} e_{0}, e_{i}\right]=i \alpha_{0} e_{i},
$$

i.e.,

$$
\Delta\left(e_{i}\right)=i \alpha_{0} e_{i}
$$

Now take an element $a_{e_{1}, e_{i}} \in W$ such that

$$
\Delta\left(e_{1}\right)=\left[a_{e_{1}, e_{i}}, e_{1}\right], \quad \Delta\left(e_{i}\right)=\left[a_{e_{1}, e_{i}}, e_{i}\right] .
$$

Then

$$
0=\Delta\left(e_{1}\right)=\left[a_{e_{1}, e_{i}}, e_{1}\right]=\left[\sum_{j \in \mathbb{Z}} \beta_{j} e_{j}, e_{1}\right]=\sum_{j \in \mathbb{Z}} \beta_{j}(1-j) e_{j},
$$

and therefore $\beta_{j}=0$ for any $j \in \mathbb{Z}, j \neq 1$. This means that $a_{e_{1}, e_{i}}=\beta_{1} e_{1}$. Hence

$$
\Delta\left(e_{i}\right)=\left[a_{e_{1}, e_{i}}, e_{i}\right]=\left[\beta_{1} e_{1}, e_{i}\right]=(1-i) \beta_{1} e_{i+1},
$$


i.e.,

$$
\Delta\left(e_{i}\right)=(1-i) \beta_{1} e_{i+1} .
$$

Taking into account that $i \neq 0,1$, and comparing (4.1) and (4.2) we obtain that $\alpha_{0}=$ $\beta_{1}=0$, i.e.,

$$
\Delta\left(e_{i}\right)=0
$$

The proof is complete.

Lemma 4.3. Let $\Delta$ be a 2-local derivation on $W$ such that $\Delta\left(e_{i}\right)=0$ for all $i \in \mathbb{Z}$. Then $\Delta \equiv 0$.

Proof. Take an arbitrary element $x=\sum_{j \in \mathbb{Z}} c_{j} e_{j}$. Let $n_{x}$ be an index such that $c_{i}=0$ for all $|i|>n_{x}$. Then $x=\sum_{j=-n_{x}}^{n_{x}} c_{j} e_{j}$. There exists an element $a_{e_{0}, x} \in W$ such that

$$
\Delta\left(e_{0}\right)=\left[a_{e_{0}, x}, e_{0}\right], \quad \Delta(x)=\left[a_{e_{0}, x}, x\right] .
$$

Since $0=\Delta\left(e_{0}\right)=\left[a_{e_{0}, x}, e_{0}\right]$, it follows that

$$
a_{e_{0}, x}=\alpha_{0} e_{0}
$$

Therefore

$$
\Delta(x)=\left[a_{e_{0}, x}, x\right]=\left[\alpha_{0} e_{0}, \sum_{j=-n_{x}}^{n_{x}} c_{j} e_{j}\right]=-\sum_{j=-n_{x}}^{n_{x}} c_{j} \alpha_{0} j e_{j} .
$$

Now take an index $n>2 n_{x}$. Let $a_{e_{n}, x} \in W$ be an element such that

$$
\Delta\left(e_{n}\right)=\left[a_{e_{n}, x}, e_{n}\right], \quad \Delta(x)=\left[a_{e_{n}, x}, x\right] .
$$

By lemma 4.2, $\Delta\left(e_{n}\right)=0$. Since

$$
0=\Delta\left(e_{n}\right)=\left[a_{e_{n}, x}, e_{n}\right]
$$

it follows that

$$
a_{e_{n}, x}=\alpha_{n} e_{n}
$$

Then

$$
\Delta(x)=\left[a_{e_{n}, x}, x\right]=\sum_{i=-n_{x}}^{n_{x}}\left[\alpha_{n} e_{n}, c_{i} e_{i}\right]=\sum_{-n_{x}}^{n_{x}}(n-i) \alpha_{n} c_{i} e_{n+i} .
$$

Since $n>2 n_{x}$, it follows that $n+i>n_{x}$ for all $i \in\left\{-n_{x}, \ldots, n_{x}\right\}$. Thus comparing (4.3) and (4.4) we obtain that $\alpha_{n}=0$. This means that $\Delta(x)=0$. The proof is complete.

Now we are in position to prove Theorem 4.1

Proof of Theorem 4.1 Let $\Delta$ be a 2-local derivation on $W$. Take a derivation $D_{e_{0}, e_{1}}$ such that

$$
\Delta\left(e_{0}\right)=D_{e_{0}, e_{1}}\left(e_{0}\right) \text { and } \Delta\left(e_{1}\right)=D_{e_{0}, e_{1}}\left(e_{1}\right) \text {. }
$$

Set $\Delta_{1}=\Delta-D_{e_{0}, e_{1}}$. Then $\Delta_{1}$ is a 2-local derivation such that $\Delta_{1}\left(e_{0}\right)=\Delta_{1}\left(e_{1}\right)=0$. By lemma 4.2, $\Delta_{1}\left(e_{i}\right)=0$ for all $i \in \mathbb{Z}$. By lemma 4.3, it follows that $\Delta_{1} \equiv 0$. Thus $\Delta=D_{e_{0}, e_{1}}$ is a derivation. The proof is complete. 


\subsection{2-Local derivations on the positive Witt algebra.}

Theorem 4.4. Any 2-local derivation on $W^{+}$is a derivation.

For the proof of this Theorem we need several Lemmas.

Lemma 4.5. Let $\Delta$ be a 2-local derivation on $W^{+}$such that

$$
\Delta\left(e_{1}\right)=\Delta\left(e_{2}\right)=0 .
$$

Then $\Delta\left(e_{j}\right)=0$ for all $j \in \mathbb{N}$.

Proof. By the definition of 2-local derivations, we can find a derivation $D_{1}$ such that

$$
\Delta\left(e_{1}\right)=D_{1}\left(e_{1}\right), \quad \Delta\left(e_{j}\right)=D_{1}\left(e_{j}\right) .
$$

Then

$$
0=\Delta\left(e_{1}\right)=D_{1}\left(e_{1}\right)=a_{1} e_{1}+\sum_{i \geq 3}(2-i) a_{i} e_{i}
$$

and therefore

$$
a_{i}=0, \quad i \neq 2 \text {. }
$$

Thus

$$
\Delta\left(e_{j}\right)=D_{1}\left(e_{j}\right)=(j-1) a_{2} e_{j+1}, \quad j \geq 3 .
$$

Now take a derivation $D_{2}$ such that

$$
\Delta\left(e_{2}\right)=D_{2}\left(e_{2}\right)=0, \quad \Delta\left(e_{j}\right)=D_{2}\left(e_{j}\right) .
$$

Thus

$$
0=\Delta\left(e_{2}\right)=D_{2}\left(e_{2}\right)=2 a_{1}^{(2)} e_{2}+a_{2}^{(2)} e_{3}+\sum_{i \geq 4}(3-i) a_{i}^{(2)} e_{i+1},
$$

i.e.,

This means that

$$
a_{i}^{(2)}=0, \quad i \neq 3
$$

$$
\Delta\left(e_{j}\right)=(j-2) a_{3}^{(2)} e_{j+2}, \quad j \geq 3 .
$$

Comparing (4.5) and (4.6) we obtain that

$$
(j-1) a_{2} e_{j+1}=(j-2) a_{3}^{(2)} e_{j+2}, \quad j \geq 3 .
$$

Thus $a_{2}=0$, and therefore $a_{i}=0$ for all $i$. So $\Delta\left(e_{j}\right)=0, j \geq 3$. The proof is complete.

Lemma 4.6. Let $\Delta\left(e_{i}\right)=0$ for all $i \in \mathbb{N}$. Then $\Delta \equiv 0$.

Proof. Let $x=\sum_{k=1}^{n} x_{k} e_{k}$ be a non zero element from $W^{+}$. By (3.1) there exists an element $a_{e_{1}, x} \in W^{+}+\left\langle e_{0}\right\rangle$ such that $\Delta\left(e_{1}\right)=\left[a_{e_{1}, x}, e_{1}\right]$ and $\Delta(x)=\left[a_{e_{1}, x}, e_{1}\right]$. Since $\Delta\left(e_{1}\right)=0$, it follows that $a_{e_{1}, x}=\alpha_{1}^{(1)} e_{1}$. Then

$$
\Delta(x)=\left[a_{e_{1}, x}, x\right]=\alpha_{1}^{(1)} \sum_{k=2}^{n}(k-1) x_{k} e_{k+1} .
$$

Now take an number $m$ such that $m>2 n$. Again by (3.1) there exists an element $a_{e_{m}, x} \in W^{+}+\left\langle e_{0}\right\rangle$ such that $\Delta\left(e_{m}\right)=\left[a_{e_{m}, x}, e_{m}\right]$ and $\Delta(x)=\left[a_{e_{m}, x}, e_{m}\right]$. Since $\Delta\left(e_{m}\right)=$ 0 , it follows that $a_{e_{m}, x}=\alpha_{m}^{(m)} e_{m}$. Then

$$
\Delta(x)=\alpha_{e_{m}, x}^{(m)} \sum_{k=1}^{n}(k-m) x_{k} e_{k+m} .
$$


Taking into account that $m>2 n$, and comparing the right sides of the equalities (4.7) and (4.8), we obtain that $\alpha_{e_{m}, x}^{(m)}=0$. Thus $\Delta(x)=0$. The proof is complete.

Now we are in position to prove Theorem 4.4

Proof of Theorem 4.4 Let $\Delta$ be a 2-local derivation on $W^{+}$. Take a derivation $D_{e_{1}, e_{2}}$ such that

$$
\Delta\left(e_{1}\right)=D_{e_{1}, e_{2}}\left(e_{1}\right) \text { and } \Delta\left(e_{2}\right)=D_{e_{1}, e_{2}}\left(e_{2}\right) .
$$

Set $\Delta_{1}=\Delta-D_{e_{1}, e_{2}}$. Then $\Delta_{1}$ is a 2-local derivation such that $\Delta_{1}\left(e_{1}\right)=\Delta_{1}\left(e_{2}\right)=0$. By lemma 4.5, $\Delta_{1}\left(e_{i}\right)=0$ for all $i \in \mathbb{N}$. By lemma 4.6, it follows that $\Delta_{1} \equiv 0$. Thus $\Delta=D_{e_{1}, e_{2}}$ is a derivation. The proof is complete.

4.3. An example of a 2-local derivation which is not a derivation on an infinite-dimensional Lie algebra. Let us consider the following (see [5]) so-called thin Lie algebra $\mathcal{L}$ with a basis $\left\{e_{n}: n \in \mathbb{N}\right\}$, which is defined by the following table of multiplications of the basis elements:

$$
\left[e_{1}, e_{n}\right]=e_{n+1}, \quad n \geq 2 .
$$

and other products of the basis elements being zero.

Theorem 4.7. Any derivation $D$ on the algebra $\mathcal{L}$ has the following form:

$$
\begin{aligned}
& D\left(e_{1}\right)=\sum_{i=1}^{n} \alpha_{i} e_{i}, \\
& D\left(e_{2}\right)=\sum_{i=2}^{n} \beta_{i} e_{i}, \\
& D\left(e_{j}\right)=\left((j-2) \alpha_{1}+\beta_{2}\right) e_{j}+\sum_{i=1}^{n} \beta_{i+2} e_{i+j}, \quad j \geq 3,
\end{aligned}
$$

where $\alpha_{i}, \beta_{i} \in \mathbb{C}, i=1, \ldots, n$, and $n \in \mathbb{N}$.

Proof. Let $D$ be a derivation on $\mathcal{L}$. We set

$$
D\left(e_{1}\right)=\sum_{i=1}^{n} \alpha_{i} e_{i}, \quad D\left(e_{2}\right)=\sum_{i=1}^{n} \beta_{i} e_{i} .
$$

We have

$$
\begin{aligned}
D\left(e_{3}\right) & =D\left(\left[e_{1}, e_{2}\right]\right)=\left[D\left(e_{1}\right), e_{2}\right]+\left[e_{1}, D\left(e_{2}\right)\right]= \\
& =\left[\sum_{i=1}^{n} \alpha_{i} e_{i}, e_{2}\right]+\left[e_{1}, \sum_{i=1}^{n} \beta_{i} e_{i}\right]= \\
& =\left(\alpha_{1}+\beta_{2}\right) e_{3}+\sum_{i=1}^{n} \beta_{i+2} e_{i+3} .
\end{aligned}
$$

Using $\left[e_{2}, e_{3}\right]=0$, we have

$$
\begin{aligned}
0=D\left(\left[e_{2}, e_{3}\right]\right) & =\left[D\left(e_{2}\right), e_{3}\right]+\left[e_{2}, D\left(e_{3}\right)\right]=\left[\sum_{i=1}^{n} \beta_{i} e_{i}, e_{3}\right]+ \\
& +\left[e_{2},\left(\alpha_{1}+\beta_{2}\right) e_{3}+\sum_{i=1}^{n} \beta_{i+2} e_{i+3}\right]=\beta_{1} e_{4},
\end{aligned}
$$


Thus $\beta_{1}=0$. Further

$$
\begin{aligned}
D\left(e_{4}\right) & =D\left(\left[e_{1}, e_{3}\right]\right)=\left[D\left(e_{1}\right), e_{3}\right]+\left[e_{1}, D\left(e_{3}\right)\right]= \\
& =\left[\sum_{i=1}^{n} \alpha_{i} e_{i}, e_{3}\right]+\left[e_{1},\left(\alpha_{1}+\beta_{2}\right) e_{3}+\sum_{i=1}^{n} \beta_{i+2} e_{i+3}\right]= \\
& =\left(2 \alpha_{1}+\beta_{2}\right) e_{4}+\sum_{i=1}^{n} \beta_{i+2} e_{i+4} .
\end{aligned}
$$

With similar arguments applied to the products $\left[e_{1}, e_{j}\right]=e_{j+1}$ and by the induction on $j$, it is easy to check that the following identities hold for $j \geq 3$ :

$$
D\left(e_{j}\right)=\left((j-2) \alpha_{1}+\beta_{2}\right) e_{j}+\sum_{i=1}^{n} \beta_{i+2} e_{i+j}, \quad j \geq 3 .
$$

The proof is complete.

Theorem 4.8. Let $\mathcal{L}$ be the thin Lie algebra. Then $\mathcal{L}$ admits a 2-local derivation which is not a derivation.

Proof. For $x=\sum_{i=1}^{n} x_{i} e_{i} \in \mathcal{L}$ set

$$
\Delta(x)= \begin{cases}0, & \text { if } x_{1}=0 \\ \sum_{i=2}^{n} x_{i} e_{i}, & \text { if } x_{1} \neq 0 .\end{cases}
$$

We shall show that $\Delta$ is a 2-local derivation on $\mathcal{L}$, which is not a derivation.

Firstly, we show that $\Delta$ is not a derivation. Take the elements $x=e_{1}+e_{2}$ and $y=-e_{1}+e_{2}$. We have

and

$$
\Delta(x+y)=\Delta\left(2 e_{2}\right)=0
$$

Thus

$$
\Delta(x)+\Delta(y)=\Delta\left(e_{1}+e_{2}\right)+\Delta\left(-e_{1}+e_{2}\right)=2 e_{2}
$$

$$
\Delta(x+y) \neq \Delta(x)+\Delta(y)
$$

So, $\Delta$ is not additive, and therefore is not a derivation.

Let us consider the linear maps $D_{1}$ and $D_{2}$ on $\mathcal{L}$ defined as:

$$
D_{1}\left(e_{n}\right)= \begin{cases}\sum_{k=2}^{m} \alpha_{k} e_{k}, & \text { if } n=1, \\ 0, & \text { if } n \geq 2,\end{cases}
$$

where $\alpha_{k} \in \mathbb{C}, k=2, \ldots, m$, and $m \in \mathbb{N}$ and

$$
D_{2}\left(e_{n}\right)= \begin{cases}0, & \text { if } n=1 \\ e_{n}, & \text { if } n \geq 2 .\end{cases}
$$

By (4.9), it follows that both $D_{1}$ and $D_{2}$ are derivations on $\mathcal{L}$. that

For any $x=\sum_{k=1}^{n_{x}} x_{k} e_{k}, y=\sum_{k=1}^{n_{y}} y_{k} e_{k} \in \mathcal{L}$ we need to find a derivation $D=D_{x, y}$ such

$$
\Delta(x)=D(x) \text { and } \Delta(y)=D(y) .
$$

It suffices to consider the following three cases.

Case 1. Let $x_{1}=y_{1}=0$. In this case, we take $D \equiv 0$, because $\Delta(x)=\Delta(y)=0$. 
Case 2. Let $x_{1}=0, y_{1} \neq 0$. In this case we take the derivation $D_{1}$ of the form (4.10) with $\alpha_{1}=0, \alpha_{k}=\frac{y_{k}}{y_{1}}, 2 \leq k \leq n_{y}$. Then

$$
\Delta(x)=0=D_{1}(x)
$$

and

$$
\Delta(y)=\sum_{k=2}^{n_{y}} y_{k} e_{k}=y_{1} \sum_{k=2}^{n_{y}} \frac{y_{k}}{y_{1}} e_{k}=D_{1}(y) .
$$

So, $D$ is a derivation such that $\Delta(x)=D(x), \Delta(y)=D(y)$.

Case 3. Let $x_{1} \neq 0, y_{1} \neq 0$. In this case we take the derivation $D_{2}$ of the form (4.11). Then

and

$$
\Delta(x)=\sum_{k=2}^{n_{x}} x_{k} e_{k}=D_{2}(x)
$$

$$
\Delta(y)=\sum_{k=2}^{n_{y}} y_{k} e_{k}=D_{2}(y)
$$

Therefore in all cases we constructed a derivation on $\mathcal{L}$ such that $\Delta(x)=$ $D(x), \Delta(y)=D(y)$, i.e. $\Delta$ is a 2-local derivation which is not a derivation. The proof is complete.

\section{REFERENCES}

[1] Ayupov Sh.A., Kudaybergenov K.K., 2-Local automorphisms on finite dimensional Lie algebras, Linear Algebra and its Applications, 507 , 121-131 (2016).

[2] Ayupov Sh.A., Kudaybergenov K.K., Rakhimov I.S., 2-Local derivations on finite-dimensional Lie algebras, Linear Algebra and its Applications, 474, 1-11 (2015).

[3] Chen Z., Wang D., 2-Local automorphisms of finite-dimensional simple Lie algebras, Linear Algebra and its Applications, 486, 335-344 (2015).

[4] Kac V., Raina A., Bombay lectures on highest weight representations of infinite-dimensional Lie algebras, World Sci.Singapore, 1987.

[5] Khakimdjanova K., Khakimdjanov Yu., Sur une classe d'algebres de Lie de dimension infinie, Comm. Algebra, Vol.29(1), 177-191 (2001).

[6] Ikeda T., Kawamoto N., On the derivations of generalized Witt algebras over a field of characteristic zero, Hiroshima Math.J., 20, 47-55 (1990).

[7] Millionshchikov D.V., Naturally graded Lie algebras (Carnot algebras) of slow growth, Mat. Sb., Forthcoming paper (Mi msb9055)

[8] Šemrl P., Local automorphisms and derivations on $B(H)$, Proc. Amer. Math. Soc., 125, 2677-2680 (1997).

1 V.I.Romanovskiy Institute of Mathematics, Uzbekistan Academy of Sciences, 81, Mirzo UlughbeK Street, 100170, Tashkent, UzBekistan

${ }^{2}$ National University of Uzbekistan, 4, University street, 100174, Tashkent, UzbeKISTAN

E-mail address: sh_ayupov@mail.ru

${ }^{3}$ National University of Uzbekistan, 4, University street, 100174, Tashkent, UzBeKISTAN

E-mail address: baxtiyor_yusupov_93@mail.ru 